\title{
Questões de gênero e raça no contexto das organizações: um mapeamento da produção científica do EnEO e EnGPR no período de 2000 a 2019
}

\author{
Gender and race issues in the context of organizations: a mapping of the scientific production of
}

EnEO and EnGPR in the period 2000 to 2019

Cuestiones de género y raza en el contexto de las organizaciones: un mapeo de la producción científica de EnEO y EnGPR en el período 2000 a 2019

Recebido: 26/01/2022 | Revisado: 30/01/2022 | Aceito: 01/02/2022 | Publicado: 03/02/2022

\author{
Isabel Bruna Correia de Brito \\ ORCID: https://orcid.org/0000-0002-6087-862X \\ Universidade Federal Rural do Semi-Árido, Brasil \\ E-mail: isabelbcbrito@gmail.com \\ Luciana Holanda Nepomuceno \\ ORCID: https://orcid.org/0000-0002-5386-0800 \\ Universidade Federal Rural do Semi-Árido, Brasil \\ E-mail: luciananepomuceno@gmail.com \\ Fábio Chaves Nobre \\ ORCID: https://orcid.org/0000-0001-9011-4252 \\ Universidade Federal Rural do Semi-Árido, Brasil \\ E-mail: fabio.nobre@ufersa.edu.br
}

\begin{abstract}
Resumo
Este artigo explora o acervo de publicações da Associação Nacional de Pós Graduação em Administração (ANPAD), especificamente nos eventos Encontro Nacional de Estudos Organizações (EnEO) e Encontro de Gestão de Pessoas e Relações de Trabalho (EnGPR), identificando os artigos que versam sobre questões de gênero e relações raciais no contexto organizacional. Tendo como objetivo analisar as principais características das publicações sobre os referidos temas, a metodologia utilizada qualifica a pesquisa como bibliográfica e descritiva com uso de VOSviewer. Os resultados encontrados indicam que os artigos apresentam uma forte prevalência de trabalhos sobre gênero, com abordagem das questões relativas à desigualdade e descrição das dificuldades vividas pelas mulheres ao desempenhar papéis historicamente destinado aos homens, como liderança e prática empreendedora. A análise das palavras-chave e dos objetivos das pesquisas converge com esta leitura. A distribuição das publicações ao longo do tempo identifica um aspecto fragmentário, não sugerindo fortalecimento das pesquisas no campo. Quanto aos aspectos metodológicos, as publicações seguem as tendências do campo das pesquisas no campo dos estudos organizacionais, apresentando uma grande maioria de estudos qualitativos, com emprego de entrevistas e/ou estudo de caso como técnica de coleta de dados e destaque para análise de conteúdo na discussão dos resultados.
\end{abstract}

Palavras-chave: Gênero; Raça; Estudos organizacionais e administrativos.

\begin{abstract}
This work explores the collection of publications of the National Association of Graduate Studies in Administration (ANPAD), specifically in the events National Meeting of Organizational Studies (EnEO) and Meeting of People Management and Labor Relations (EnGPR), identifying the articles that deal with gender issues and race relations in the organizational context. Aiming to analyze the main characteristics of publications on these topics, the methodology used qualifies the research as bibliographic and descriptive using VOSviewer. The results found indicate that the articles present a strong prevalence of works on gender, addressing issues related to inequality and describing the difficulties experienced by women when performing roles historically destined for men, such as leadership and entrepreneurial practice. The analysis of keywords and research objectives converges with this reading. The distribution of publications over time identifies a fragmentary aspect, not suggesting a strengthening of research in the field. As for the methodological aspects, the publications follow trends in the field of research in the field of organizational studies, presenting a large majority of qualitative studies, using interviews and/or case studies as a data collection technique and highlighting content analysis. in the discussion of the results.
\end{abstract}

Keywords: Gender; Race; Organizational and administrative studies.

\section{Resumen}

Este artículo explora el acervo de publicaciones de la Asociación Nacional de Posgrados en Administración (ANPAD), específicamente en los eventos Encuentro Nacional de Estudios Organizacionales (EnEO) y Encuentro de 
Gestión de Personas y Relaciones Laborales (EnGPR), identificando los artículos que tratan cuestiones de género y relaciones raciales en el contexto organizacional. Con el objetivo de analizar las principales características de las publicaciones sobre estos temas, la metodología utilizada califica la investigación como bibliográfica y descriptiva utilizando VOSviewer. Los resultados encontrados indican que los artículos presentan una fuerte prevalencia de trabajos sobre género, abordando cuestiones relacionadas con la desigualdad y describiendo las dificultades experimentadas por las mujeres al desempeñar roles históricamente destinados a los hombres, como el liderazgo y la práctica empresarial. El análisis de palabras clave y objetivos de investigación converge con esta lectura. La distribución de las publicaciones a lo largo del tiempo identifica un aspecto fragmentario, no sugiriendo un fortalecimiento de la investigación en el campo. En cuanto a los aspectos metodológicos, las publicaciones siguen tendencias en el campo de la investigación en el campo de los estudios organizacionales, presentando una gran mayoría de estudios cualitativos, utilizando entrevistas y/o estudios de casos como técnica de recolección de datos y destacando el análisis de contenido en la discusión. de los resultados.

Palabras clave: Género; Raza; Estudios organizacionales y administrativos.

\section{Introdução}

Os estudos que utilizam as categorias gênero e raça, na teoria organizacional e administrativa (TOA), ainda são incipientes, apesar de sistematicamente vir sendo assinalados como um campo a ser explorado (Ferreira et al., 2015). Andrade et al. (2014) indicam um aumento gradativo no número de publicações sobre a temática gênero no campo dos estudos organizacionais, porém destacam que se tratava de uma área ainda em construção. Aparentemente encontram ainda menos visibilidade na construção de conhecimento em TOA, pesquisas que envolvam a categoria raça ou estudos interseccionais que mobilizem as duas categorias, raça e gênero.

Há pouco mais de uma década, os estudos de Vieira e Caldas (2005) ressaltavam a falta de estudos sobre raça e racismo nas organizações brasileiras e as pesquisas de Conceição (2009) demonstravam e questionavam a ausência da raçaetnia como tema de pesquisa nos estudos organizacionais. Proni e Gomes (2015), em seu estudo sobre precariedade ocupacional, reforçam essas constatações afirmando que a discriminação racial e a desigualdade de gênero são costumeiramente desconsideradas nas análises sobre os fatores responsáveis pelo quadro conjuntural do mercado de trabalho nacional.

A partir da compreensão deste cenário e compreendendo a relevância de acompanhar os rumos da produção científica em TOA, propôs-se este trabalho que explorou o acervo de publicações da Associação Nacional de Pós Graduação em Administração (ANPAD), especificamente nos eventos Encontro Nacional de Estudos Organizacionais (EnEO) e Encontro de Gestão de Pessoas e Relações de Trabalho (EnGPR), identificando os artigos que versam sobre questões de gênero e/ou relações raciais no contexto organizacional. Busca-se, através de análises das principais características das publicações que tratam das questões de gênero e/ou raça, não só descrever as características identificadas, mas compreender como está sendo construído o campo de estudo sobre os temas em TOA.

\section{Referencial Teórico}

\subsection{Questões raciais nos estudos organizacionais}

Apesar de as questões raciais estarem em crescente surgimento nas pesquisas científicas, escassa produção acadêmica brasileira é produzida em prol desse assunto relevante para sociedade nas áreas de administração e estudos organizacionais (Conceição, 2009; Costa \& Ferreira, 2006; Coelho Junior \& Hein, 2021). Sabendo-se que as estruturas organizacionais são influenciadas pela cultura da sociedade e, de forma concomitante, as dinâmicas organizacionais sustentam e moldam as relações sociais, justifica-se a preocupação e análise acerca da carência dessas publicações. Aplica-se ainda o conhecimento de que a maioria dos estudiosos se encontra na alta sociedade, assim, dificilmente abrangem sua visão para os problemas presentes no país (Conceição, 2009).

No que tange o conceito de raça, entende-se que esta se trata de uma construção simbólica, ou seja, diz respeito à uma 
origem comum, podendo ser caracterizada por aspectos físicos, corporais, sociais e psicológicos (Conceição, 2009; Rosa, 2014). Apesar de ser um fenômeno complexo, a construção da raça entrelaça-se com as relações de dominação e divisão social, construídas socialmente parar hierarquizar os povos (Rosa, 2014).

Costa e Ferreira (2006) explicam a relação entre a raça e condição social. Entende-se que o racismo se apresenta por meio de uma construção histórica e social de relação de subordinação pelos brancos, assim, desde a escravidão foram negadas oportunidades para as pessoas negras, ou seja, o preconceito intrínseco na sociedade impossibilitou ao negro o acesso à educação de qualidade e condições mínimas para uma vida decente que deveriam ser permitidas para todo cidadão, consequentemente dificultou a busca por emprego e por cargos mais altos, além de bons salários. Não obstante, essas condições ainda privaram o acesso às estruturas acadêmicas e, por conseguinte a realização de pesquisas e produção de conhecimento, não somente científico.

A concepção do racismo indireto ou racismo institucional é um aspecto relevante para compreender as desigualdades presentes dentro das estruturas organizacionais, tendo em vista que nem sempre o racismo é notório de forma explícita e reproduzido de forma consciente (Lopez, 2012). Assim, essa ideia explica que o racismo enraizado nas organizações é fruto da desigualdade racial brasileira, que atua por meio de construções sociais como a invalidação de oportunidades igualitárias, desde a contratação de funcionários de diferentes raças até a distribuição de benefícios como promoções e aumento no salário. Dessa maneira, o conhecimento desse termo pode auxiliar na criação de propostas que possibilitem a luta contra a discriminação racial (Conceição, 2009).

\subsection{As dificuldades das mulheres em cargos de liderança}

Em contexto histórico, no que diz respeito a visão do trabalho como divisão social, a mulher foi moldada para ser mãe e esposa, e essa seria unicamente sua obrigação, o que tornava um emprego inviável para condição de mulher (Macêdo \& Macêdo, 2004; Texeira, 2008). A partir da Revolução Industrial, quando foi permitido às mulheres o acesso aos postos de trabalho, não the foram concedidos o "reconhecimento, nem as oportunidades de acesso a determinados cargos e nem a ascensão profissional dada aos homens" (Magalhães \& Paula, 2016; Lima et al., 2009).

A inserção da mulher no mercado de trabalho foi crescente ao longo dos anos (Baylão \& Schettino, 2014). As posições hierárquicas antes ocupadas por mulheres jovens, solteiras e sem filhos, passaram a serem ocupadas também por mulheres mais velhas, mães e casadas (Azevedo, 2007; Miranda et al., 2011). Apesar de estarem ativas no mercado de trabalho, os cargos ocupados pelas mulheres no contexto organizacional são, em sua maioria, posições subalternas aos homens, marcadas pela divisão sexual do trabalho, a qual apresenta diversas desigualdades (Silva \& Silva, 2010; Lima et al., 2009; Santos \& Antunes, 2011). Miranda et al. (2011) indicam que apesar do avanço das mulheres em assumirem cargos de poder, estas têm assumido mais cargos denominados masculinos do que os homens têm assumido cargos tipicamente femininos. Neste contexto, Marques et al. (2021) apontam a necessidade do uso do "gênero" como categoria analítica relevante nos estudos relacionados ao mercado de trabalho e economia, incluindo uma perspectiva que a teoria tradicional não alcança.

A escolaridade das mulheres tem se destacado por ultrapassar o nível da educação masculina mas, apesar disso, esse fator pouco influencia na escalada para uma posição de maior poder no nível hierárquico das organizações (Lima et al., 2009). Miranda et al. (2011) apontam o evento chamado "teto de vidro", este seria uma barreira que intensifica a dificuldade de ascensão das mulheres nos cargos destinados aos homens. Os autores acrescentam ainda que evolução feminina nas posições de poder não acontecem de forma linear, uma vez que a maior parte dos cargos de liderança é ocupado por meio de indicação vinda dos superiores, ou seja, de homens para homens.

Ubal et al. (2019) discorrem sobre a respeito da diferença entre estilo de liderança feminino e masculino, uma vez que o primeiro é visto como o fator denominado "comum", este seria constituído ações passivas, dotado de sensibilidade e carisma, 
ao contrário da liderança masculina, denominado agentic, composta de atitudes de dominação e superioridade (características essas tipicamente associadas aos homens). Em consonância, sob uma ótica machista, a mulher é vista como ser dotado de sentimentalismo e guiado pelas intuições, assim, para subir nos cargos hierárquicos das organizações, seria necessário adotar uma postura máscula, mas sem abandonar sua feminilidade (Lima et al., 2009).

\subsection{Mulheres negras em posições de poder}

A condição de vulnerabilidade social da mulher negra não é um fenômeno recente e engloba um conjunto de indicadores que apontam a desvantagem que elas vivenciam para acessar direitos sociais fundamentais (Vieira, 2017). Santos, Beretta e Antoniassi Júnior (2021) apontam que a atual conjuntura da mulher negra no Brasil ainda apresenta significativos resquícios da estrutura e da cultura da sociedade escravocrata do país. Entender a historicidade e a atualidade deste processo permite compreender porque as mulheres negras são mais frequentemente a parcela da população submetida a trabalhos degradantes, com baixo prestigio social e a dificuldade que as mesmas encontram para atingir posições de poder, liderança e prestígio nas organizações (Crenshaw, 2004).

Para Oliveira e Both (2013), a mulher negra sofre duplamente durante sua jornada profissional, tanto devido ao patriarcado, como ao racismo inerente à sociedade e às organizações. A pesquisa da Fundação Perseu Abramo (2010), indica que a concepção de trabalho feminino como força de trabalho secundária atua de forma persistente no imaginário, inclusive na formulação de políticas públicas, sustentada e mantendo uma ideia de família nuclear e heteronormativa cujo papel do homem seria o de provedor e a mulher é entendida como mera cuidadora. Para as mulheres negras a situação se agrava posto que o cenário colonial que ainda se faz presente na contemporaneidade brasileira propicia um acirramento nos níveis de exploração a que estão submetidas as pessoas negras e, mesmo no contexto do trabalho assalariado, as desigualdades de raça e gênero relacionam-se e mantem desigualdades de classe, circunscrevendo o horizonte das mulheres negras a atividades ligadas às tarefas do lar e do cuidado (Carneiro \& Santos, 1985, p. 15).

Assim, no que tange as desvantagens que circundam as mulheres negras, muitas variáveis podem aparentemente justificar sua posição precária no mercado de trabalho como, por exemplo, a baixa escolaridade (Oliveira \& Both, 2013; Vieira, 2017), entretanto é importante perceber este fenômeno de forma interseccional e, assim, entender que a mulher negra passa por diversas adversidades para avançar no desenvolvimento de suas habilidades, inserir-se no mercado de trabalho e ocupar cargos de liderança na hierarquia de uma organização e que tais adversidades são determinadas menos por qualquer questão individual específica e mais pela forma como operam o racismo institucional e o machismo estrutural (Oliveira \& Both, 2017; Crenshaw, 2004).

\section{Metodologia}

Para Eco (1983), "organizar uma bibliografia significa buscar aquilo cuja existência ainda se ignora” (p. 42). Em termos de resultados, a pesquisa bibliográfica permite: (a) fazer um histórico sobre o tema; (b) atualizar-se sobre o tema escolhido; (c) formular problemas e questões; (d) levantar contradições sobre o tema; e (e) evitar a repetição de trabalhos já realizados (Eco, 1983). Considerando as questões expostas, a pesquisa bibliográfica permite que o tema das relações raciais estabeleça um novo diálogo com os estudos organizacionais ao mesmo tempo em que chama atenção para as possíveis lacunas de pesquisa.

A metodologia de um trabalho é o caminho para o alcance do objetivo de qualquer estudo científico. Cruz e Medeiros (2021) indicam que ao procurar resposta para determinada indagação em um processo de investigação científica, é preciso seguir critérios na definição da metodologia a fim de conferir maior confiabilidade, como a utilização de procedimentos racionais e sistemáticos. Cervo e Bervian (1996, p. 62) apontam que "método ou metodologia é a ordem que se deve impor aos 
diferentes processos necessários para atingir um fim dado ou um resultado desejado". Neste caso, esta pesquisa é classificada, quanto aos fins, como uma pesquisa descritiva porque visa analisar as características da produção científica atual sobre questões de gênero e relações raciais no contexto organizacional, em consonância com a proposição de Vergara (2009, p. 42) que define a pesquisa descritiva como aquela que "expõe as características de determinada população ou de determinado fenômeno". Quanto aos meios é classificada como uma pesquisa bibliográfica já que realizada a partir do levantamento de referências teóricas já analisadas e publicadas.

Este trabalho foi realizado utilizando as técnicas de bibliometria visando o mapeamento da produção acadêmica sobre questões de gênero e relações raciais nas organizações, explorando o acervo de dois congressos da ANPAD - Associação Nacional de Pós-Graduação e Pesquisa em Administração, sendo estes o EnEO - Encontro de Estudos Organizacionais e o EnGPR - Encontro de Gestão de Pessoas e Relações de Trabalho.

O levantamento em questão foi composto pelas versões completas dos artigos que se enquadravam nos temas de interesse, selecionados e apresentados entre o ano de 2000 a 2019 (EnEO) e de 2007 a 2017 (EnGPR). O critério para a seleção do material foi que o artigo apresentasse um discurso relativo à diversidade racial e de gênero nas organizações, além da abordagem da liderança e do empreendedorismo feminino.

As palavras-chaves escolhidas para fins de seleção dos artigos dos congressos foram definidas através da proximidade com o tema de gênero e raças nas organizações. Sendo estas as escolhidas: Desigualdade de gênero; Diversidade; Empreendedorismo feminino; Gênero; Mulher; Mulher empreendedora; Mulher executiva; Preconceito; Raça; Relações de gênero; Trabalho familiar.

Esta coleta - que indicou a existência de 129 trabalhos, sendo 86 publicados no EnEO e 43 encontrados no EnGPR foi feita por meio do portal da Anpad, onde são disponibilizados todos os trabalhos. Foram selecionados os que tratavam diretamente de questões de gênero e/ou relações raciais no contexto organizacional. Após uma análise dos resumos de cada artigo, 17 foram descartados devido a não correspondência com o tema em questão, restando 112 artigos para serem analisados e trabalhados - sendo destes 69 encontrados no EnEO e 43 publicados no EnGPR. O gráfico 1 mostra quantos artigos foram identificados em cada ano por congresso.

Através do software Mendeley, os artigos dos eventos EnEO e EnGPR foram reunidos e salvos em um arquivo do tipo ris, que posteriormente foi utilizado no software VOSviewer para visualizar a rede de dados das palavras-chaves selecionadas, presentes nos 112 artigos escolhidos. Primeiro, ao abrir o VOSviewer, selecionou-se a opção de criar, para que pudéssemos gerar um mapa, e logo após escolheu-se a opção de criar um mapa baseado nos dados bibliográficos, inseriu-se o arquivo ris, e, em seguida, o tipo de análise escolhida foi a de ocorrência, selecionando, também, a opção de análise completa das palavraschaves. O mínimo de ocorrências escolhido por palavras foram 2. Gerando, assim, um mapa de 43 palavras. O mapa é uma rede que liga todos os itens de interesse, nesse caso as palavras-chaves, e os links, que são a conexão entre elas. 
Gráfico 1 - Artigos encontrados por ano no Encontro de Estudos Organizacionais da ANPAD e Encontro de Gestão de Pessoas e Relações de Trabalho da ANPAD.

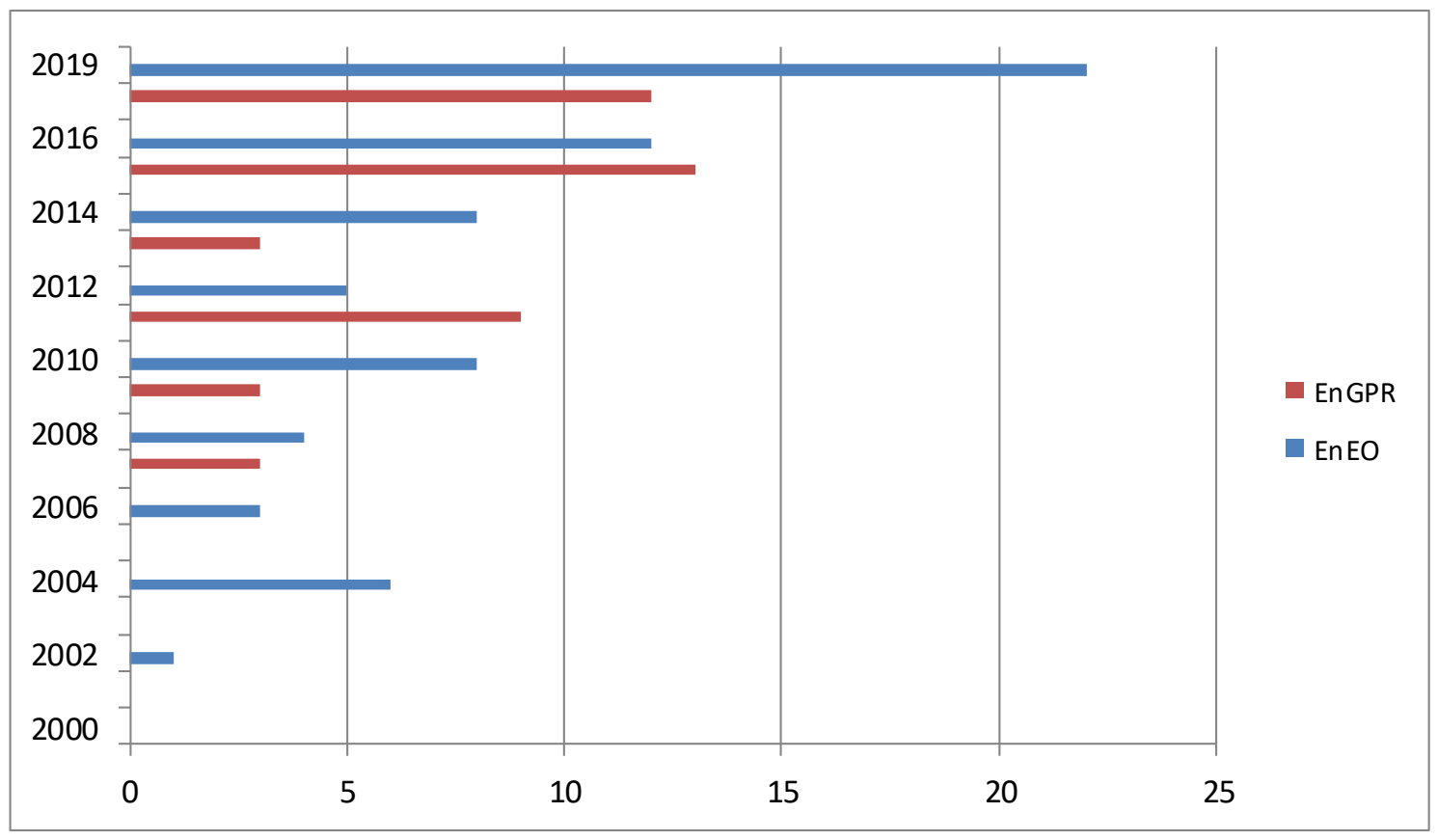

Fonte: Dados da Pesquisa (2021).

Referente ao Gráfico 1, os artigos foram separados por ano e por evento onde foi publicado antes da realização de leitura sistemática de cada um deles. Posteriormente, identificou-se os temas principais dos artigos a partir do título e do objetivo. Na coleta de dados, também foi realizada a classificação dos artigos quanto a metodologia, para identificar o tipo e as estratégias de pesquisa e as técnicas de coleta de dados e as técnicas de análise de dados utilizadas. Estas informações foram arquivadas em um banco de dados montado em uma planilha eletrônica, contendo, além disso, as seguintes características de todas as publicações: títulos, palavras-chave, resumos, autores, instituições. Posteriormente os dados também foram tratados por meio do software VOSviewer.

\section{Resultados e Discussão}

Este trabalho se propõe a descrever e analisar as características apresentados pelos estudos sobre raça e gênero, no âmbito da TOA, publicados em eventos significativos na área. Para atingir este objetivo, as características analisadas foram divididas quanto a: tema e subtema dos trabalhos, análise das palavras-chave e objetivos das pesquisas, distribuição no tempo e aspectos metodológicos. Entretanto, antes da discussão proposta, é preciso destacar que a discrepância da produção nos dois eventos, com o Encontro de Estudos Organizacionais apresentando mais trabalhos sobre raça/gênero/diversidade que o Encontro de Gestão de Pessoas e Relações de Trabalho, pode ser creditada, em grande parte, à quantidade de edições de cada evento, já que o EnGPR só teve seu início em 2007 e, no período determinado para este estudo, o EnEO já tinha tido quatro edições com 10 artigos publicados relativos aos temas discutidos aqui.

Especificamente no que se refere às características dos trabalhos pesquisados, na análise dos temas abordados nos artigos, encontra-se significativa predominância de estudos focados em gênero (85) em comparação com os artigos sobre raça (10) ou que tratam dos temas de maneira interseccional ou incluídos no conceito mais amplo de diversidade (17). A pouca visibilidade que as questões de diversidade racial encontram nos estudos organizacionais e administrativos já foi identificado previamente nas pesquisas de Vieira e Caldas (2005) que apontaram a falta de estudos sobre raça e racismo nas organizações 
brasileiras, de Conceição (2009) que aprofunda o debate e questiona a ausência de investigações sobre raça-etnia neste campo e de Rosa (2014) que o discute o tema das relações raciais no Brasil e explora suas interfaces com os estudos sobre gestão da diversidade nas organizações

Destaca-se que a grande maioria dos textos, ao tratar de gênero, volta-se para questões relativas à relação das mulheres com o mundo organizacional e do trabalho (Capelle et al., 2006; Eccel \& Grisci, 2011). Tal característica discursiva aponta a naturalização do espaço laboral e organizacional como próprio do masculino. A presença, a atuação, as características gerenciais das práticas empreendidas por homens não são tomadas como questões, problematizadas ou qualificadas como questões de gênero, mas como universais. Entre os 112 trabalhos encontrados, apenas quatro tratam especificamente as questões do masculino como questões de gênero, o artigo de Matos et al. (2014) que discute a possibilidade da atividade empreendedora subverter as normas de gênero; o texto de Medeiros e Silva (2014) que trata do apelo a um ideal de masculinidade para incentivar o consumo de carne; a pesquisa de Eccel e Grisci (2010), em uma empresa petroquímica e que teve como objetivo descrever a figura do homem engenheiro como representante do ideal de masculinidade, e o artigo de Moura (2019) que discutiu a masculinidade tóxica e como esse modelo afeta a vida dos homens gays.

Os trabalhos que discutem raça no contexto organizacional o fazem relacionando quase automaticamente a questão às pessoas negras, em um movimento muito semelhante ao presente nos textos sobre gênero, apagando o processo de racialização hegemônico das pessoas lidas como brancas e silenciando sobre outros grupos racializados e marginalizados, como os grupos de ascendência indígena. Um texto que se destaca por não reproduzir acriticamente essa lógica é o artigo teórico de Teixeira e Oliveira (2016) que se propõe a discutir o próprio conceito de raça e a pertinência do uso desta categoria no âmbito dos estudos organizacionais.

Os trabalhos que se voltam para o tema diversidade, publicados no EnEo, tratam o tema de forma crítica, apontando o caráter ideológico do mesmo e a forma manipulativa e ainda discriminatória com que a gestão da diversidade se faz presente no cotidiano das organizações. Nos trabalhos publicados no EnGPR que abordam a diversidade nas organizações, a tendência é focar em estudos descritivos sobre as políticas e práticas gerenciais e sobre as habilidades requeridas ao gestor para trabalhar com as questões de diversidade no cotidiano das organizações. Entretanto é no EnGPR que se encontra o único estudo que explicitamente se propõe interseccional, uma investigação empírica focada em gênero e raça e como esses fatores influenciam o acesso ao mercado de trabalho das mulheres haitianas (Pereira \& Oliveira, 2017).

A Figura 1 apresenta o mapa de visualização de rede, representando a teia de relações entre as palavras-chave dos artigos pesquisados. As cores dizem respeito aos cinco conjuntos de clusters formados, identificados na Tabela 1. Os artigos que tratam de gênero relacionam esse tema com gestão, mulher, organizações, mercado de trabalho, liderança, cultura organizacional, desigualdade de gênero, mulher executiva, raça, minorias, diversidade, trabalho, identidade, carreira, agronegócio, empoderamento, empreendedorismo feminino, empreendedorismo, violência simbólica, socialização organizacional, identidade profissional, interseccionalidade, relações de poder, feminino, trabalho-família, mobilidade, classe, pós-estruturalismo, trabalho familiar, relações de gênero e mulheres. Os artigos pesquisados, que tratam de raça, relacionam esse tema com trabalho, gênero, diversidade, organizações, minorias, interseccionalidade, estudos organizacionais e classe. E os artigos que tratam de diversidade relacionam com gênero, raça, trabalho, minorias, organizações de trabalho, mulher executiva, liderança, mercado de trabalho, organizações, gestão, mulher, gestão da diversidade, pós-estruturalismo, políticas, práticas, gestores, estudos organizacionais e identidade. 
Figura 1: Mapa de visualização da rede das palavras-chave.

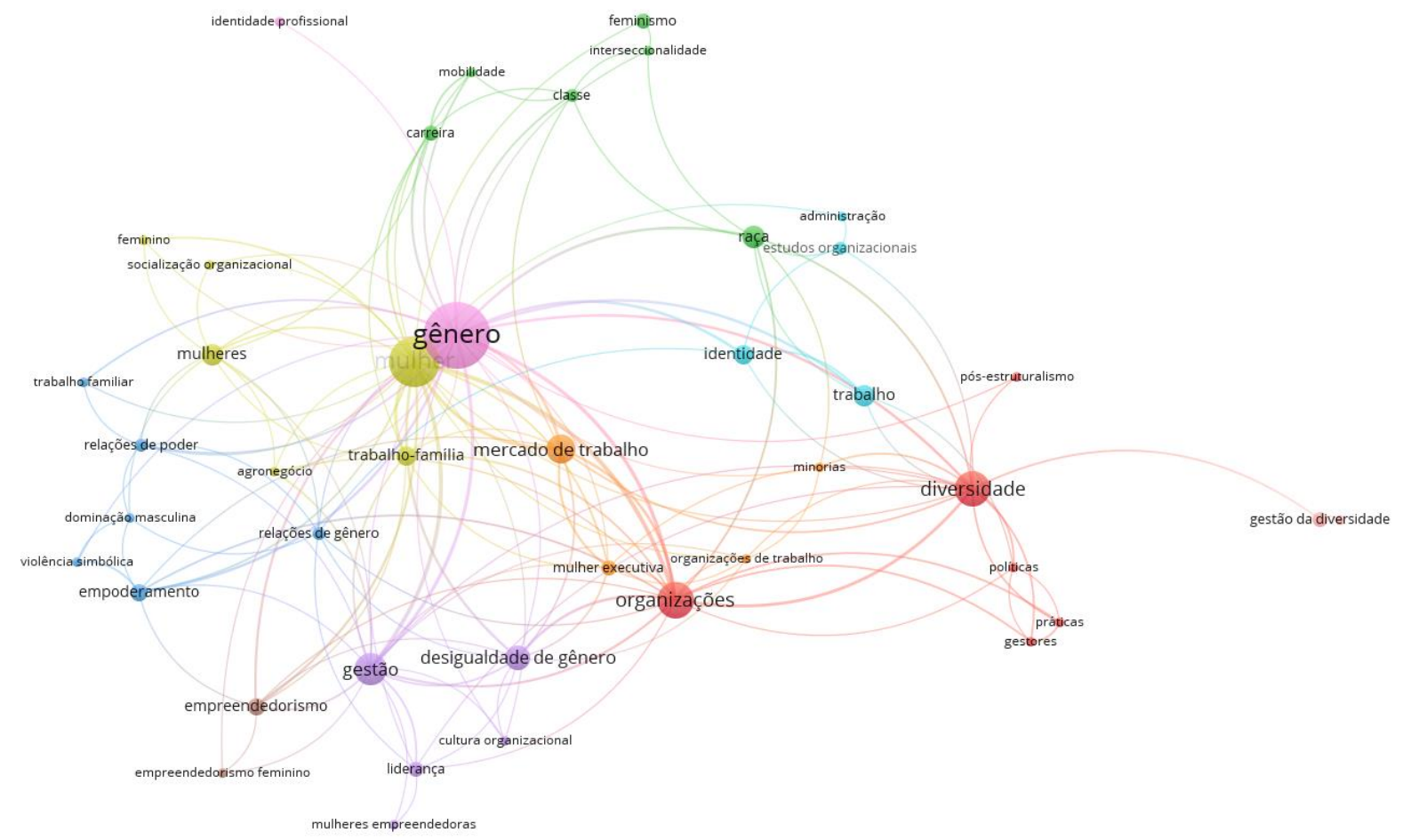

Fonte: Dados da Pesquisa (2021).

A análise dos sub-temas, ou seja, a abordagem que é dada aos temas gênero, raça e diversidade e ao que são relacionados, nos artigos publicados nos eventos, parece confirmar o que a apreciação inicial havia indicado, por exemplo, a ênfase em uma abordagem instrumental do tema gênero, sendo tratado com um olhar reprodutivista dos aspectos encarados como neutros de uma gestão dominada por homens, em artigos que versam sobre desempenho, práticas gerenciais, empreendedorismo, sem uma discussão significativa sobre a concepção de gênero, a diferença - se há - entre gênero e sexo e uma certa tendência à naturalização e biologização das diferenças comportamentais. Já as poucas produções que apresentam as questões raciais impulsionando o debate ainda se restringem, primordialmente, às questões de estereótipos e discriminação. A análise da constituição dos clusters esclarece ainda mais essa análise.

A Tabela 1 apresenta os conjuntos de palavras - clusters - o número de conexões entre elas, a força total dessas ligações e o número de ocorrência dessas palavras, ou seja, a quantidade de vezes em que elas aparecem nos artigos. O total de clusters formados foram 10. Nota-se que a palavra que possui o maior número de ligações é "gênero", possuindo 31 ligações, força total de ligações totalizando 81, e 45 ocorrências. As palavras com maior número de ligações em cada cluster são, respectivamente: Organizações (em um cluster formado em torno do tema diversidade); Raça (no cluster que versa sobre Interseccionalidade); Empoderamento e Relações de Gênero (no cluster sobre desigualdade); Mulher (no cluster que trata sobre questões de gênero e o equilíbrio trabalho/família); Gestão (no cluster em que as questões de liderança feminina se destacam); Identidade e Trabalho (no cluster que compreende as discussões sobre identidade no âmbito dos Estudos Organizacionais); Mulher executiva e Mercado de Trabalho (no cluster sobre Minorias e Mercado de Trabalho); Empreendedorismo (no cluster sobre empreendedorismo feminino); Gênero (em um cluster que trata especificamente desse tema) e Gestão da Diversidade (em um cluster que relaciona o tema com análise fílmica). 
Research, Society and Development, v. 11, n. 2, e49311226187, 2022

(CC BY 4.0) | ISSN 2525-3409 | DOI: http://dx.doi.org/10.33448/rsd-v11i2.26187

Tabela 1: Conjuntos de clusters gerados através do VOSviewer.

\begin{tabular}{|c|c|c|c|c|}
\hline Cluster & Palavras & Links & Total Link Strength & Ocorrência \\
\hline \multirow{6}{*}{$\begin{array}{c}\text { Cluster } 1 \text { - A diversidade } \\
\text { nas organizações de } \\
\text { trabalho }\end{array}$} & Diversidade & 18 & 32 & 16 \\
\hline & Gestores & 4 & 7 & 2 \\
\hline & Organizações & 18 & 50 & 17 \\
\hline & Políticas & 5 & 6 & 2 \\
\hline & Práticas & 4 & 7 & 2 \\
\hline & Pós-estruturalismo & 2 & 2 & 2 \\
\hline \multirow{6}{*}{$\begin{array}{c}\text { Cluster } 2 \text { - } \\
\text { Interseccionalidade na } \\
\text { carreira feminina }\end{array}$} & Carreira & 6 & 10 & 4 \\
\hline & Classe & 6 & 7 & 3 \\
\hline & Feminismo & 2 & 2 & 4 \\
\hline & Interseccionalidade & 4 & 4 & 2 \\
\hline & Mobilidade & 4 & 6 & 2 \\
\hline & Raça & 8 & 13 & 8 \\
\hline \multirow{6}{*}{$\begin{array}{c}\text { Cluster } 3 \text { - Gestão e } \\
\text { desigualdade de gênero }\end{array}$} & Dominação masculina & 5 & 5 & 2 \\
\hline & Empoderamento & 8 & 14 & 5 \\
\hline & Relações de gênero & 8 & 8 & 3 \\
\hline & Relações de poder & 7 & 8 & 3 \\
\hline & Trabalho familiar & 3 & 4 & 2 \\
\hline & Violência simbólica & 3 & 4 & 2 \\
\hline \multirow{6}{*}{$\begin{array}{l}\text { Cluster } 4 \text { - Mulheres e a } \\
\text { relação de trabalho- } \\
\text { família }\end{array}$} & Agronegócio & 5 & 6 & 2 \\
\hline & Feminino & 3 & 4 & 2 \\
\hline & Mulher & 22 & 68 & 29 \\
\hline & Mulheres & 11 & 13 & 7 \\
\hline & Socialização organizacional & 3 & 3 & 2 \\
\hline & Trabalho-Família & 9 & 16 & 6 \\
\hline \multirow{4}{*}{$\begin{array}{c}\text { Cluster } 5 \text { - Liderança } \\
\text { feminina nas } \\
\text { organizações }\end{array}$} & Cultura organizacional & 4 & 4 & 2 \\
\hline & Desigualdade de gênero & 11 & 20 & 9 \\
\hline & Gestão & 17 & 36 & 14 \\
\hline & Liderança & 7 & 8 & 4 \\
\hline \multirow{5}{*}{$\begin{array}{c}\text { Cluster } 6 \text { - A identidade } \\
\text { nos estudos } \\
\text { organizacionais }\end{array}$} & Mulheres empreendedoras & 2 & 2 & 2 \\
\hline & Administração & 2 & 2 & 2 \\
\hline & Estudos organizacionais & 4 & 4 & 3 \\
\hline & Identidade & 5 & 9 & 6 \\
\hline & Trabalho & 5 & 9 & 7 \\
\hline \multirow{4}{*}{$\begin{array}{l}\text { Cluster } 7 \text { - Minorias e as } \\
\text { organizações de trabalho }\end{array}$} & Mercado de trabalho & 9 & 25 & 12 \\
\hline & Minorias & 4 & 5 & 2 \\
\hline & Mulher executiva & 9 & 14 & 4 \\
\hline & Organizações de trabalho & 7 & 7 & 2 \\
\hline \multirow{2}{*}{$\begin{array}{c}\text { Cluster 8 - } \\
\text { Empreendedorismo } \\
\text { feminino }\end{array}$} & Empreendedorismo & 8 & 13 & 5 \\
\hline & Empreendedorismo feminino & 3 & 3 & 2 \\
\hline \multirow{2}{*}{$\begin{array}{l}\text { Cluster } 9 \text { - Gênero e } \\
\text { identidade profissional }\end{array}$} & Gênero & 31 & 81 & 45 \\
\hline & Identidade profissional & 1 & 1 & 2 \\
\hline \multirow{2}{*}{$\begin{array}{l}\text { Cluster } 10 \text { - Diversidade } \\
\text { e Análise fílmica }\end{array}$} & Análise fílmica & 1 & 1 & 2 \\
\hline & Gestão da diversidade & 2 & 3 & 4 \\
\hline
\end{tabular}

Fonte: Dados da Pesquisa (2021).

Ainda sobre a Tabela 1, destaca-se o cerne das discussões que estruturam a constituição de cada cluster. O cluster 1 - 
a diversidade nas organizações de trabalho - reúne os artigos que trabalham as políticas e práticas de gestão relacionadas ao trabalho com minorias no cotidiano organizacional. O cluster 2 - interseccionalidade na carreira feminina - se volta para os pontos de convergência e relações entre raça, gênero, classe e seu efeito na carreira e mobilidade no trabalho. O cluster 3 gestão e desigualdades de gênero - aborda, principalmente, os desafios das mulheres em posições de poder, com ênfase nas questões de relações de pode e dominação masculina. O cluster 4 - mulheres e relação trabalho/família - inclui os artigos que trabalham as dificuldades da mulher na construção de novos papéis sociais e as demandas que lhe são apresentadas de performance em cada aspecto da vida. Há, também, neste cluster, um destaque para os estudos realizados junto ao agronegócio. O cluster 5 - Liderança feminina nas organizações - envolve os aspectos da desigualdade de gênero nos cargos de liderança com destaque para o papel da cultura organizacional neste processo. O cluster 6 - a identidade nos estudos organizacionais compreende os trabalhos que discutem como as questões identitárias emergem nos trabalhos da área. O cluster 7 - minorias e as organizações de trabalho - é revelador porque apesar de tratar do tema minorias tem como destaque as questões de gênero em detrimento aos demais sub-temas como raça, classe, orientação sexual. O cluster 8 - empreendedorismo feminino - diz respeito a supostas características específicas de uma prática empreendendora realizada por mulheres. O cluster 9 - Gênero e identidade profissional - é o que engloba o tema de maior força e o conteúdo dos trabalhos explicita a relação tendenciosa entre tratar as questões de gênero como questões do feminino. Por fim, o cluster 10 - Diversidade e Análise - compreende os trabalhos que fazem suas proposições a partir de reflexões sobre produções áudio-visuais.

A Figura 2 mostra esse desenvolvimento dos temas e subtemas por ano de publicação, é possível visualizar a frequência de ocorrência das palavras em cada período, ressaltando que a maior frequência é vista a partir de 2010 em diante, bem como apresenta o Gráfico 1.

Figura 2: Mapa de visualização de sobreposição.

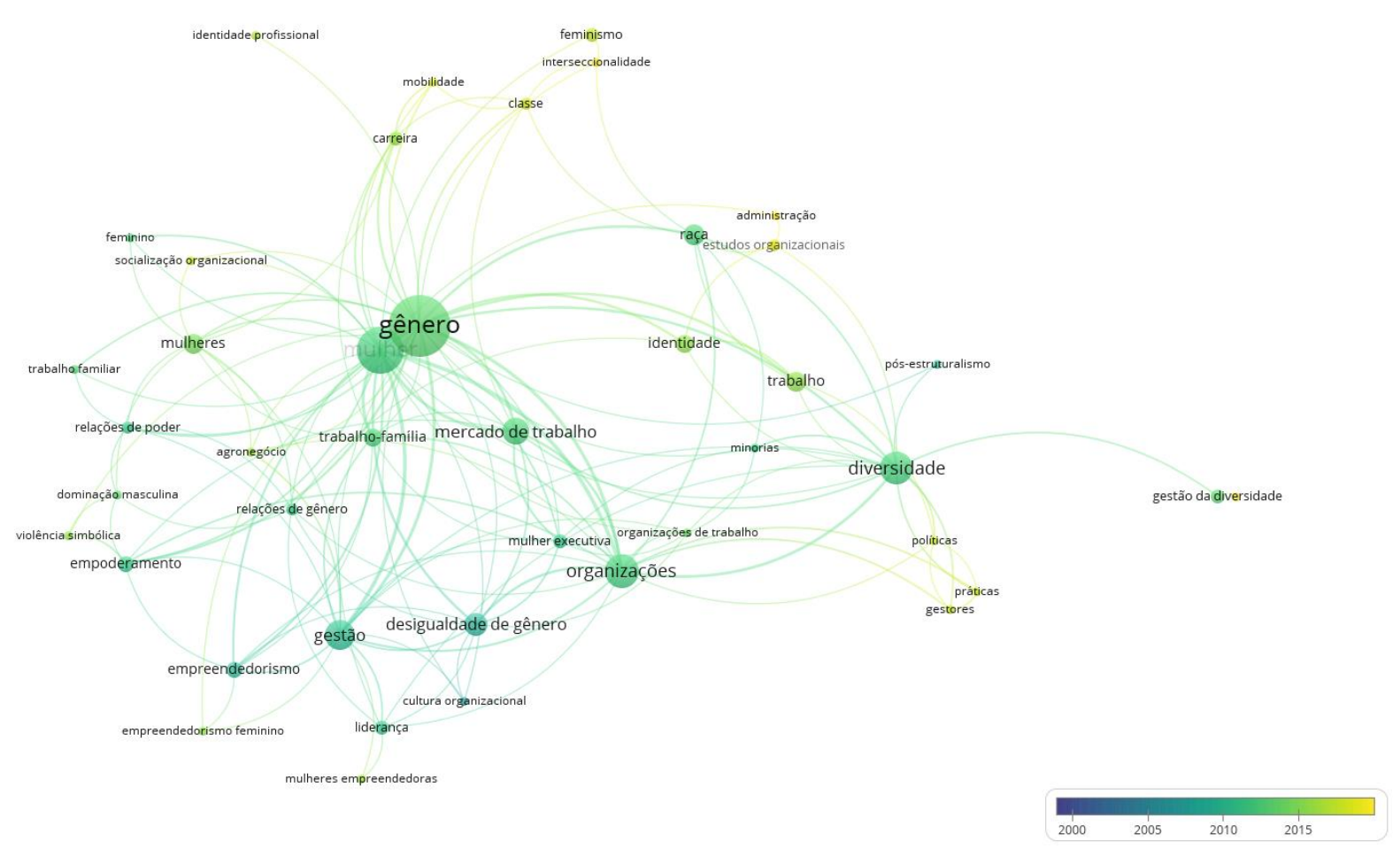

Fonte: Dados da Pesquisa (2021).

Aprofundando-se o olhar, percebe-se que apesar dos temas de gênero e raça, além das questões tratadas de forma ampla como diversidade, estarem constantemente abordados nos eventos estudados, uma certa fragmentação parece indicar 
que não há indícios de um fortalecimento da pesquisa nestas áreas nem mesmo o desenvolvimento de focos ou busca de uma abordagem específica. Sobre os estudos que trabalham a questão de raça, por exemplo, se por um lado o aspecto quantitativo poderia indicar alguma consistência já que 7 dos 10 artigos foram publicados entre 2016 e 2019 e apenas 3 entre 2000 e 2015 ; a análise interna dos mesmos não parece sustentar esta conclusão, não se observa aumento de consistência, integração ou relação entre as produções, os três artigos publicados em 2019 (Santos \& Oliveira, 2019), por exemplo, não trazem nas referências bibliográficas nenhum dos artigos sobre o tema anteriormente publicados no EnEO ou EnGPR. Também nos subtemas percebe-se a estagnação ou falta de aprofundamento dos recortes, os estudos sobre gênero e empreendedorismo, por exemplo, apresentam uma publicação por ano nos eventos de 2004, 2008, 2011, 2014, 2015 e 2019 e duas publicações em 2010. Observe-se que o artigo mais recente (Ubal et al. 2019) parece ecoar as questões do mais antigo (Rodrigues \& Santos, 2004), discutindo as principais dificuldades encontradas pelas mulheres ao exercer papel de liderança nas organizações.

Em relação aos aspectos metodológicos, os 112 trabalhos pesquisados, apresentam-se preferencialmente como qualitativas com apenas 8 estudos identificados como quantitativos e 3 classificados como um estudo quali-quanti. Os estudos quantitativos utilizaram preferencialmente questionários como técnica de coleta de dados e, quanto às técnicas de análise de dados, seguiram uma técnica primária com o uso de estatística descritiva. Apenas dois artigos aprofundaram a análise utilizando análise fatorial (Vieira et al., 2016a; Vieira et al., 2016b), ambos encabeçados pela mesma pesquisadora.

De maneira geral, evidencia-se a predominância de estudos de caso e entrevistas como técnica de coleta de dados (Gráfico 2); e uso destacado de análise de conteúdo e análise do discurso, no que tange às técnicas de análise de dados (Gráfico 3), conforme gráficos abaixo:

Gráfico 2: Técnicas de Coleta de Dados.

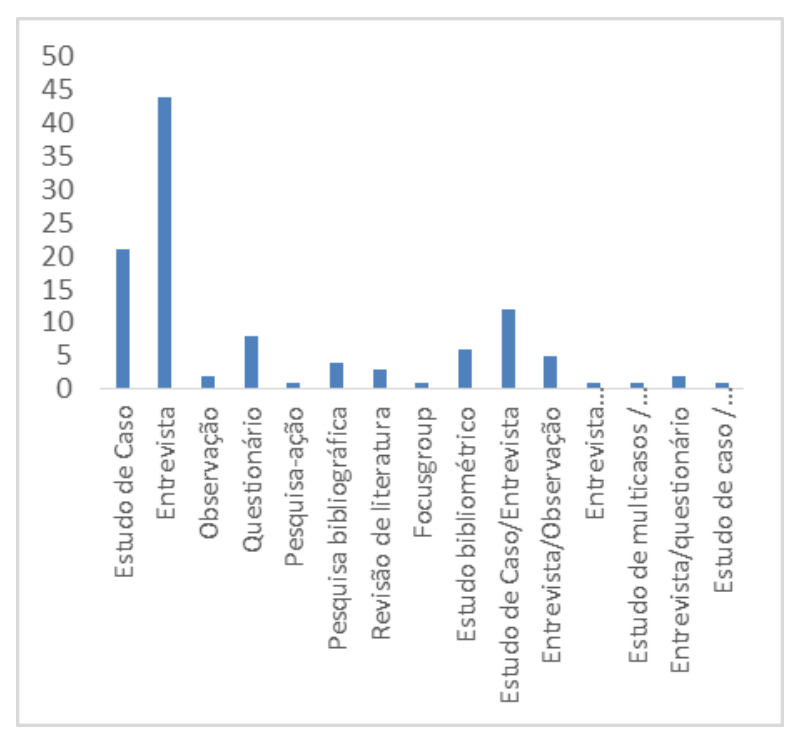

Fonte: Dados da Pesquisa (2021). 
Gráfico 3: Análise de dados.

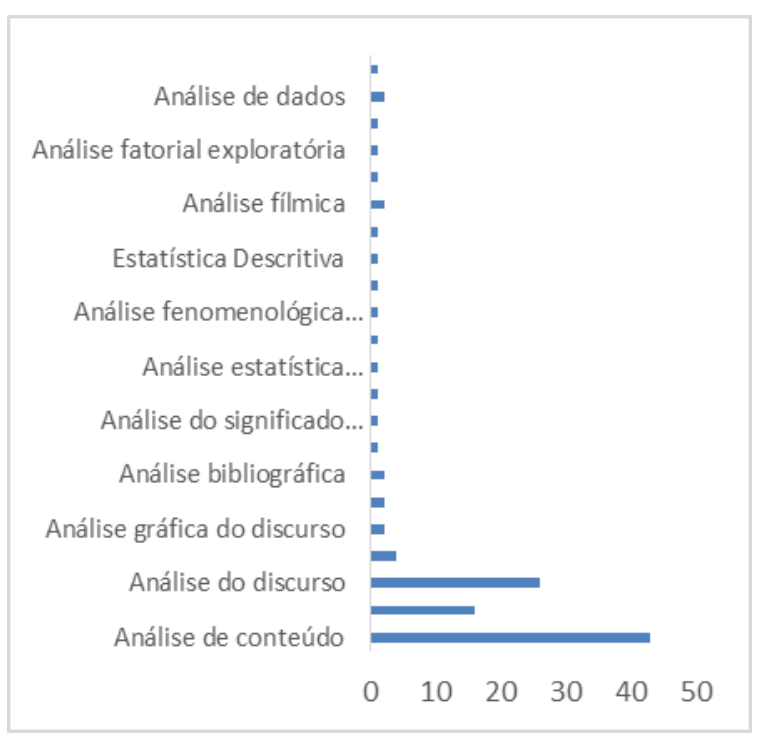

Fonte: Dados da Pesquisa (2021).

A respeito do Gráfico 2, ressalta-se que entre 112 trabalhos publicados, 65 informam utilizar entrevista ou estudo de caso como técnica de coleta de dados e mais 12 trabalhos indicaram usar as duas técnicas de forma combinada, destacando essas técnicas, de maneira incisiva, frente as demais. No uso da técnica da entrevista, ressalta-se a relevância da entrevista semi-estruturada, tendo destaque em 36 pesquisas, sozinha ou combinada com outros recursos. Tal destaque é coerente e se deve, como se pode perceber, à predominância dessas técnicas nos mais variados estudos qualitativos (Taquette \& Borges, 2020; Godoi, Bandeira-De-Mello \& Silva, 2012; Minayo, 2010). O gráfico 3 apresenta a análise de conteúdo como a análise mais utilizada nas pesquisas encontradas a respeito dos temas de gênero, raça e diversidade. Este resultado converge com os estudos de Bastos et al. (2019) que apontam a forte presença desta técnica nas publicações da área de administração indexadas na plataforma Scielo. Essa tendência também se evidencia em trabalhos com recortes específicos no campo da administração, como nos achados de Teixeira et al. (2013) que, ao pesquisarem sobre estudos em administração que utilizavam a triangulação metodológica, identificaram que entre os métodos qualitativos de análise utilizados, a análise de conteúdo se apresentava em 49\% dos artigos e de Soares et al. (2011) que identificaram que a análise de conteúdo foi a técnica mais utilizada pelos autores que publicaram sobre a área de Administração Pública. 
Figura 3: Campos de estudos de gênero e raça nas organizações.

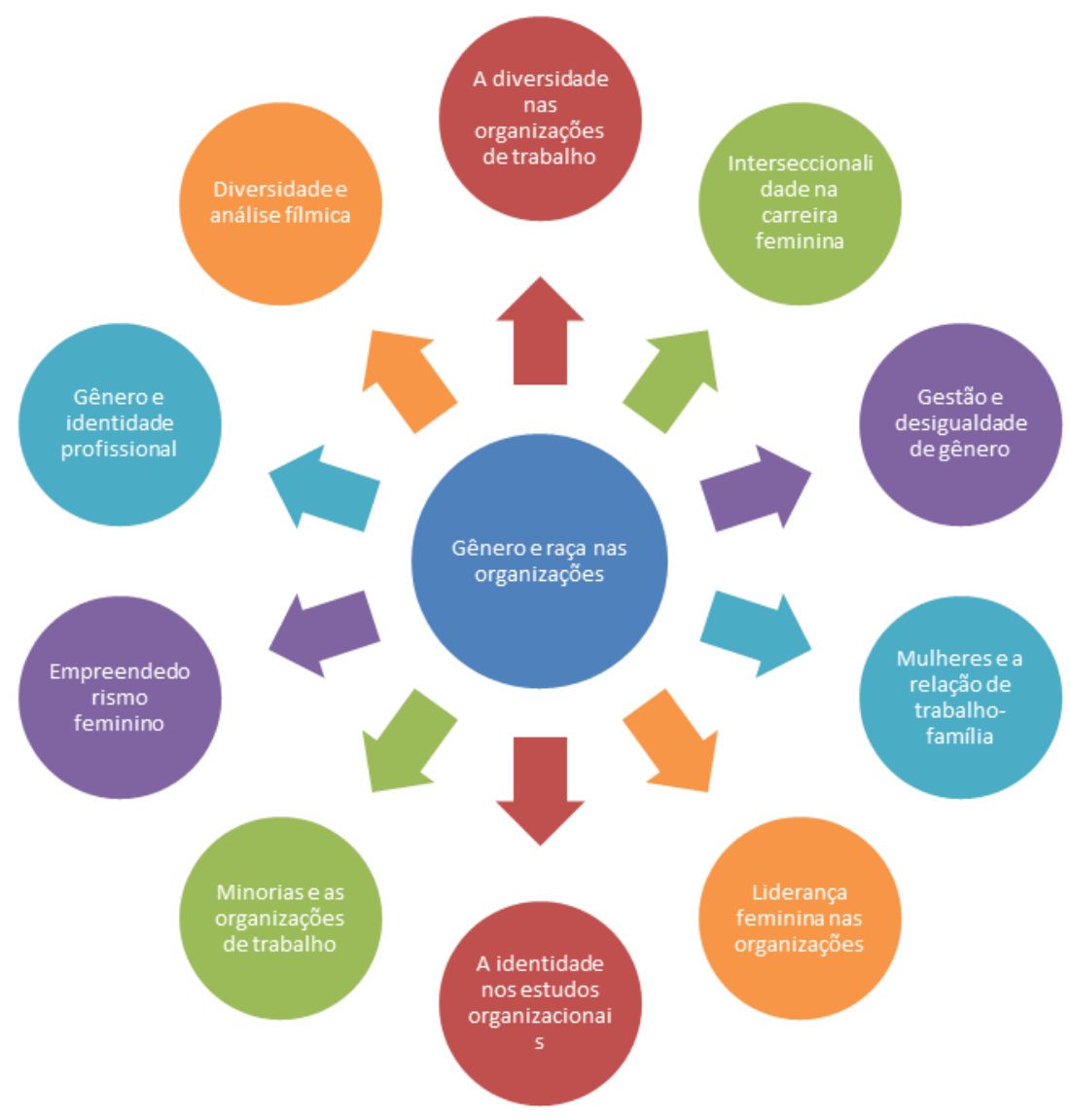

Fonte: Dados da Pesquisa (2021).

Os resultados desta investigação sobre as características dos estudos publicados nos eventos EnEO e EnGPR, no período de 2001 a 2019, sobre gênero, raça e diversidade, indicam que quanto aos temas e subtemas (presentes na Figura 3), os artigos apresentam uma forte prevalência de trabalhos sobre gênero, com uma abordagem das questões relativas à desigualdade no âmbito das organizações e na descrição das dificuldades vividas pelas mulheres ao desempenhar papéis historicamente destinado aos homens, como a liderança nas empresas e a prática empreendedora. A análise das palavras-chave e dos objetivos das pesquisas converge com esta leitura sobre o conteúdo dos artigos. Uma análise que se detém na distribuição das publicações ao longo do tempo sugere um aspecto fragmentário e disperso destes estudos, não indicando um fortalecimento das pesquisas nestes campos. Quanto aos aspectos metodológicos, este trabalho identificou que as publicações estudadas seguem as tendências no campo das pesquisas no campo dos estudos organizacionais, apresentando uma grande maioria de estudos qualitativos, com emprego de entrevistas e/ou estudo de caso como técnica de coleta de dados e destaque para análise de conteúdo e interpretativista no tratamento e discussão dos dados obtidos.

\section{Considerações Finais}

As minorias, em sua diversidade, têm lutado por sua inserção, crescimento e visibilidade, direitos e transformações no mercado de trabalho, assim como nos demais âmbitos da sociedade, e tal processo ecoar nas produções acadêmicas é um efeito esperado e evidenciado neste e em outros estudos que discutem o que se produz na área de estudos organizacionais e administrativos. Encontrar artigos que analisam questões sobre gênero no ambiente empresarial nas últimas décadas, por exemplo, tem sido comum (Capelle et al., 2006) e comparando o levantamento atual com as proposições de Conceição (2009), que apresentava sua inquietação ao analisar publicações acadêmicas em administração e verificar o pouco material produzido 
sobre raça, pode-se identificar avanços também na produção sobre o tema. Entretanto a literatura visitada e os dados suscitados por esta pesquisa sugerem que os estudos sobre raça, gênero e, principalmente, uma abordagem interseccional que os contemple, ainda se mostram incipientes em eventos significativos, com abrangência nacional, na área de administração e estudos organizacionais. No que se refere especificamente às questões de gênero, destaca-se o silêncio sobre masculinidade; ampliar este foco, conforme Eccel e Grisci (2009), permitiria discutir a universalidade daquele trabalhador tomado como norma e complexificar o debate, não restringindo a abordagem a um contraponto em relação ao qual mulheres e suas vivências são comparadas. Quando se complexifica o exame dos trabalhos ainda se encontra silêncio sobre questões fundamentais como os debates complementares sobre masculinidade, já referido, e branquitude, por exemplo, que poderiam ampliar compreensões, saindo da descrição imobilizante sobre desigualdade e discriminação, avançando nas proposições e entendimento dos processos de possível transformação do ambiente organizacional. Também se evidencia que é preciso avançar no que se refere a uma abordagem interseccional ao se identificar que mesmo os estudos sobre diversidade tratam as categorias gênero, classe, raça, orientação sexual, como estanques e desconectadas.

O estudo encontrou alguns obstáculos que dificultaram a realização da análise em todos os aspectos anteriormente planejados. Por exemplo, a não identificação da relação dos autores com grupos de pesquisa ou universidades de origem impediu uma discussão sobre quais os centros ou núcleos que tem papel relevante no desenvolvimento do conhecimento sobre raça e gênero na área. Outro aspecto a se considerar é a variedade de formas como se apresenta ou mesmo se nomeia os procedimentos metodológicos, às vezes omitindo informações que poderiam contribuir no entendimento mais adequado de como os estudos foram conduzidos. Apesar de se considerar os limites comuns e não desprezíveis de se publicar em congressos e eventos afins, como o tamanho ou restrições de formato, é importante destacar esse resultado, buscando o aprimoramento da comunicação acadêmica dos achados de pesquisa que fazem a área avançar.

Como uma caracterização geral do universo de estudos em dois dos principais eventos nacionais na área de Administração, os principais resultados indicam um quadro em que predominam estudos descritivos, sem uma abordagem crítica dos próprios conceitos empregados, sustentando-se na polarização entre o masculino e o feminino, nos estudos de gênero, e na racialização apenas das pessoas negras, no que tange aos estudos sobre raça. Além disso se destaca que os estudos discutidos apoiam-se na realização de investigações teórico-empíricas de natureza qualitativa, empregando o método de estudo de caso e a entrevista como técnica de coleta de dados, sendo estes, além do tratamento e interpretação via análise de conteúdo, os métodos costumeiros na área, não parecendo haver uma reflexão sobre a necessidade de um direcionamento específico para o tratamento destes campos.

Entende-se que o tratamento interseccional de categorias como raça e gênero, acompanhadas de outras tais como classe, identidade e orientação sexual, pode enriquecer a área dos estudos organizacionais e administrativos, não só ampliando o escopo dos temas tratados, dando destaque para a dinâmica das relações, os aspectos concretos e a subjetividades dos atores envolvidos, mas também permitindo avanços metodológicos, fortalecimento de linhas de pesquisa e construção de canais de comunicação e interação entre grupos de origem e abordagens diversas.

Neste sentido, acredita-se que este trabalho ofereça significativa contribuição não só pelo que apresenta ao mapear a produção mais recente sobre raça e gênero nos eventos específicos, mas pelo que ele não pôde retratar, pelo que evidenciou de silêncios e pontos a avançar na construção do conhecimento no campo.

Indica-se a continuação e aprofundamento deste caminho de pesquisa em trabalhos futuros com a ampliação da base de dados pesquisada, integrando, por exemplo, artigos publicados em outros eventos relevantes, tais como os Encontros da Associação Nacional de Pós-Graduação e Pesquisa em Administração (EnANPAD), assim como trabalhos publicados em revistas qualificadas pela CAPES. Indica-se também a possibilidade de trabalhos no campo empírico das organizações em que estas variáveis, raça e gênero, assim como variáveis de identidade e orientação sexual, possam ser tomadas como relevantes na 
análise, desnaturalizando o discurso prevalente sobre o sujeito organizacional

\section{Referências}

Andrade, L. F. S., Macedo, A. S., \& Oliveira, M. L. S. (2014). A Produção Científica em Gênero no Brasil: um panorama dos grupos de pesquisa em administração. RAM, Rev. Adm. Mackenzie. 15(6), 48-75.

Azevedo, M. C. (2007). A mulher no mercado de trabalho brasileiro. I Encontro de Gestão de Pessoas e Relações de Trabalho. Natal/RN.

Bastos, M.H.R., Oliveira, U.R., Souza, T.C.R., Santos, R.F., \& Lago, M.M. (2019). Análise de discurso e Análise de Conteúdo: Um levantamento de suas aplicações nas ciências aplicadas membros da Administração. Brazilian Journal of Development. 5(11), 26301-26322. Curitiba.

Baylão, A. L. S., \& Schettino, E. M. O. (2014). A Inserção da Mulher no Mercado de Trabalho Brasileiro. XI Simpósio de Excelência em Gestão e Tecnologia. https://www.aedb.br/seget/arquivos/artigos14/20320175.pdf.

Cappelle, M., Brito, M., Melo, M., \& Vasconcelos, K. A. (2006). Produção Científica sobre Gênero na Administração: Uma Meta-Análise. In: XXX EnANPAD. Salvador.

Cervo, A., \& Bervian, P. (1996). Metodologia científica. Makron Books.

Coelho Junior, P.J., HEIN, A.S. (2021) Gênero, raça e diversidade: trajetórias profissionais de executivas negras. Organizações \& Sociedade. 28 , $265-293$.

Conceição, E. B. da. (2009). A Negação da Raça nos Estudos Organizacionais. Anais do Encontro Nacional da Associação Nacional de Pós-Graduação e Pesquisa em Administração.

Costa, S. G., \& Ferreira, C. Da S. (2006). Diversidade e Minorias nos Estudos Organizacionais Brasileiros: Presença e Lacunas na Última Década. IV Encontro de Estudos Organizacionais da ANPAD.

Crenshaw, K. (2002). A intersecionalidade da discriminação de raça e gênero. Observatório da saúde da população negra. https://nesp.unb.br/popnegra/index.php/biblioteca/2-genero-raca-e-saude/5-a-intersecionalidade-na-discriminacao-de-raca-e-genero.

CRUZ, A. C. da S. O. da; MEDEIROS, A. F. de. (2021) Theoretical-methodological construction of a research: an analysis of the path traveled. Research, Society and Development.10(17), p. e244101724708. 10.33448/rsd-v10i17.24708. https://rsdjournal.org/index.php/rsd/article/view/24708.

Eccel, C. S., \& Grisci, C. L. I. (2010). Trabalho, Gênero e Subjetividade: A Valorização dos Engenheiros em uma Empresa do Setor Petroquímico. Encontro Nacional de Estudos Organizacionais.

Eccel, C. S., \& Grisci, C. L. I. (2011). Trabalho e Gênero: a produção de masculinidades na perspectiva de homens e mulheres. CADERNOS EBAPE. 9(1).

Eco, U. (1983). Como se faz uma tese. São Paulo: Perspectiva.

Ferreira, J. B., Sadoyama, A. S. P., Correia, A. F. C., \& Gomes, P. A. (2015). Diversidade e Gênero no Contexto Organizacional: um Estudo Bibliométrico. Revista Pensamento Contemporâneo em Administração. 9(3), 45-66.

Fundação Perseu Abramo. (2010). Pesquisa Mulheres Brasileiras e Gênero nos Espaços Público e Privado. Fundação Perseu Abramo.

Godoi, C. K., Bandeira-De-Mello, R., \& Silva, A. B. (2012). (Orgs.). Pesquisa Qualitativa em Estudos Organizacionais: Paradigmas, Estratégias e Métodos. 2. ed. Saraiva.

Lima, G. S., Lima, M. S., \& Tanure, B. (2009). Os desafios da carreira da mulher executiva no Brasil. II Encontro de Gestão de Pessoas e Relações de Trabalho. Anais. Curitiba/PR.

López, L. C. (2012). O conceito de racismo institucional: aplicações no campo da saúde. Interface - Comunicação, Saúde, Educação. 16(40), 121-34.

Magalhães, A. F., \& Paula, C. R. de A. (2016). Reflexões sobre a "Inclusão" de Minorias Sociais nas Organizações de Trabalho: Uma Análise Semiótica de uma Estratégia de Recrutamento de Pessoas do McDonald's Brasil. IX Encontro de Estudos Organizacionais da ANPAD.

Marques, L. de S., Carvalho, B. do N., Santos, Z. J. C. G. dos, Leão, A. S. R., \& Lobato, T. da C. (2021). Igualdade de gênero na participação do mercado de trabalho formal de Santarém-PA: realidade ou utopia? Research, Society and Development. 10(13). https://doi.org/10.33448/rsd-v10i13.21653

Matos, F. R. N., Figueiredo, M. D., Quezado, I., \& Mesquita, R. F. (2014). Homem com H: A norma masculina do empreendedorismo informal nas confecções de jeans de Toritama/PE. In: Encontro Nacional de Estudos Organizacionais - EnEO. Gramado/RS.

Medeiros, C. R. De O., \& Silva, N.C. (2014). Homem de Verdade: Apelo a um ideal de Masculinidade em Propagandas de Fast food. Encontro Nacional de Estudos Organizacionais - EnEO

Minayo, M. C. S. (2010). Técnicas de pesquisa: entrevista como técnica privilegiada de comunicação. O desafio do conhecimento: pesquisa qualitativa em saúde. 12. ed. São Paulo: Hucitec.

Miranda, A. R. A., Mafra, F. L. N., \& Cappelle, M. C. A. (2011). Relações de Gênero e Poder: Um Estudo Com Professoras-gerentes Em Uma Universidade Pública. III Encontro de Gestão de Pessoas e Relações de Trabalho da ANPAD.

Moraes, R. (1999) Análise de conteúdo. Revista Educação. 22(37), 7-32. 
Research, Society and Development, v. 11, n. 2, e49311226187, 2022

(CC BY 4.0) | ISSN 2525-3409 | DOI: http://dx.doi.org/10.33448/rsd-v11i2.26187

Moura, R. G. (2019). A Masculinidade Tóxica e Seus Impactos Na Vida Dos Gays Dentro das Organizações. Encontro Nacional de Estudos Organizacionais $-E n E O$.

Oliveira, J. K. De, \& Both, L. J. R. G. (2017). A mulher negra em cargos de liderança: A influência do colonialismo e do feminismo negro nas relações de trabalho da mulher negra. CAD. ESC. DIR. REL. INT. (UNIBRASIL). Curitiba/PR. 27(2), 71-91.

Pereira, F. O., \& Oliveira, J. S. de. (2017). A Intersecção entre Raça, Gênero e Imigração no Mercado de Trabalho: um Estudo com Mulheres Haitianas na Cidade de Maringá, Paraná. VI Encontro de Gestão de Pessoas e Relações de Trabalho da ANPAD. Curitiba/PR.

Proni, M. W., \& Gomes, D. C. (2015). Precariedade ocupacional: uma questão de gênero e raça. Estud. av., 29(85), 137-151.

Rosa, A. R. (2014). Relações Raciais E Estudos Organizacionais No Brasil. Revista de Administração Contemporânea - RAC. 18(3), 240-260.

Santos, E. L. S., \& Oliveira, J. S. (2019). Práticas, raça e organizações empreendedoras: um estudo com empreendedores negros na região metropolitana da cidade do Rio de Janeiro. Encontro Nacional de Estudos Organizacionais - EnEO.

Santos, J. C. S. Dos, \& Antunes, E. Di D. (2011). Relações de Gêneros e Liderança nas Organizações: Rumo a um Estilo Andrógino de Gestão. III Encontro de Gestão de Pessoas e Relações de Trabalho. João Pessoa/PB.

Santos, R. das G., Beretta , R. C. de S. ., \& Antoniassi Junior, G. (2021). Protagonismo da mulher negra: uma discussão sobre igualdade de gênero e a redução das desigualdades. Research, Society and Development. 10(15), e11101522658. https://doi.org/10.33448/rsd-v10i15.22658

Silva, F.C., \& Gomes Jr. A. B. (2019). Estrelas em Pauta: Revisitando o Protagonismo Negro na Corrida Espacial da Década de 1960. Encontro Nacional de Estudos Organizacionais - EnEO.

Silva, L. B. Da; \& Silva, W. R. (2010). Abordagens Feministas em Estudos Organizacionais e o Movimento de Mulheres na Paraíba. VI Encontro de Estudos Organizacionais da ANPAD. Florianópolis/SC.

Soares, E. B. S., Pereira, A. D. S., Suzuki, J. A., \& Emmendoerfer, M. L. (2011). Análises de dados qualitativos: intersecções e diferenças em pesquisas sobre administração pública. III Encontro de Ensino e Pesquisa em Administração e Contabilidade - EnEPQ. http://www.anpad.org.br/admin/pdf/EnEPQ261.pdf.

Taquette, S.R., \& Borges, L. (2020). Pesquisa qualitativa para todos. Vozes.

Teixeira, J. C., \& Oliveira, J. S. De. (2016). Por que ainda falarmos em raça, categoria destituída de valor biológico? IX Encontro de Estudos Organizacionais da ANPAD. Anais. Belo Horizonte.

Teixeira, J. C., Nascimento, M. C. R., Antonialli, L. M. (2013). Perfil de estudos em Administração que utilizaram triangulação metodológica: uma análise dos anais do EnANPAD de 2007 a 2011. RAUSP Management Journal. 48(4), 800-812.

Teixeira, J.C., Oliveira, J.S., Mesquita, J. S. (2019). Pode a Interseccionalidade ser Afrocentrada no Campo da Administração? Um Ensaio Teórico sobre as contribuições da Teoria Interseccional para a área de Administração. Encontro Nacional de Estudos Organizacionais - EnEO.

Texeira, M. O. (2008). Desigualdades salariais entre homens e mulheres a partir de uma abordagem de economistas feministas. Niterói. 9(1), 31-45.

Ubal, V. O., Moreira, M. G., Fleck, C F. (2019). Bossy Girl: O mercado está mesmo preparado para as mulheres na liderança? Encontro Nacional de Estudos Organizacionais - EnEO. Fortaleza/CE.

Vergara, S. C. (2009). Projetos e relatórios de pesquisa em administração. Atlas.

Vieira, A. Carrieri, A. P., Monteiro, P. R R., Roquete, F. F., Carneiro, L. C. B., \& Guerra, V. A. (2016). Âncoras de Carreira e Gênero: estudo comparativo de estudantes da área da saúde e da engenharia. IX Encontro De Estudos Organizacionais Da ANPAD.

Vieira, A. Carrieri, A. P., Monteiro, P. R R., Roquete, F. F., Carneiro, L. C. B., \& Guerra, V. A. (2016a) Diferenças de Gênero no Processo de Construção das Identidades Socioprofissionais nas Áreas da Saúde e da Engenharia. Encontro Nacional de Estudos Organizacionais - EnEO.

Vieira, A. Carrieri, A. P., Monteiro, P. R R., Roquete, F. F., Carneiro, L. C. B., \& Guerra, V. A. (2016b) Âncoras de Carreira e Gênero: Estudo Comparativo de Estudantes da Área da Saúde e da Engenharia. Encontro Nacional de Estudos Organizacionais - EnEO.

Vieira, B. (2017). Mulheres negras no mercado de trabalho brasileiro: Um balanço das políticas públicas. Seminário Internacional Fazendo Gênero 11 \& 13 th Women's Worlds Congress. Florianópolis.

Vieira, M., \& Caldas, M. (2005). Teoria crítica e pós-modernismo: principais alternativas à hegemonia funcionalista. Revista de Administração de Empresas. 46(1), 59-70. 10.1590/S0034- 75902006000100006 